\title{
LOVE WAVE DISPERSION IN HETEROGENEOUS ANISOTROPIC MEDIA*
}

\author{
DON L. A N D ER SON $†$
}

In analysis is made of Love-wave propagation in a medium composed of transversely isotropic layers. This is the kind of anisotropy which most commonly occurs at the surface of the earth, and in particular is displayed by bedded sediments. The exact boundary value problem is solved for a simple layer and extended to multilayered media by a generalization of Haskell's technique. By a suitable redefinition of parameters, it is possible to cast the anisotropic problem into isotropic form so that existing programs, tables, and graphs can be used to determine the structure of layered anisotropic media.

It can be shown that the Love-wave period equation expresses the condition of constructive interference between multireflected $S I I$ waves with directionally dependent velocities. Also, it is demonstrated analytically and numerically that a rest ricted form of the anisotropy considered here is the limit of a finely laminated solid as the laminations become much smaller than a wave length. For long wave lengths, a multilayererl structure may lie replaced by an equivalent single layer.

\section{INTRODUCTION}

The earth is generally assumed in both theory and practical application to be isotropic, or at most, to be composed of isotropic layers. Sufficiently detailed studies, however, often indicate the presence of anisotropy. 'This situation usually manifests itself in the directional dependence of seismic body waves, but there are other anomalous observations which indirectly suggest a departure from isotropy (see, for instance, White and Sengbush, 1953; Jolly, 1956; Uhrig, 1955; and Layat et al, 1961). The latter authors show that large errors in depth determinations occur in seismic exploration in the Northern Sahara if the anisotropy is neglected. Gassman (1951) has presented theoretical arguments for the existence of anisotropy in granular material.

Theoretical studies of anisotropy usually deal with limiting cases such as infinitesimal thickness and infinite wave length (Helbig, 1958; Postma, 1955; White and Angona, 1955), or with infinite thickness and infinite frequency (Stoneley, 1949). The former authors consider anisotropy to be the limiting case of a laminated solid as the laminations become infinitesimal, and Stoneley considers surface wave propagation along a halfspace.

Surface wave dispersion studies on bounded, layered, or inhomogeneous media are more com- plicated and, because of the introduction of di mensions or characteristic lengths, demand frequency information that is not available in the study of limits. Anderson (1961) studied the dispersion of Rayleigh waves in a layered anisotropic solid and Love waves in simple layered media. The complete boundary value problem for $S H$ waves in multilayered material is solved exactly in this paper.

Horizontally polarized transverse waves promise to shed a great deal of light on the phenomena of anisotropy because they do not undergo change of type upon reflection or refraction in relatively simple structures and are less affected by degree of fluid saturation than $P$ waves.

\section{NOMENCLATUR}

$$
\begin{aligned}
a_{m} & =\text { layer matrix for } m \text { th layer } \\
A & =\text { product of layer matrices } a_{n-1} a_{n-2} \cdots a_{1} \\
A_{i j} & =\text { elements of product matrix } \\
b_{m}= & a_{m} a_{m-1} \text { matrix for representative doublet } \\
& \text { in a laminated medium } \\
c & =\text { phase velocity }=\omega / k \\
d & =\text { thickness of an individual layer } \\
d^{\prime} & =\text { pseudo-thickness }=(N / L)^{1 / 2} d \\
e_{i j} & =\text { elastic strains } \\
k & =\text { horizontal wave number } \\
m, n, l & =\text { direction cosines from } z \text {-axis } \\
u, v, w & =\text { displacements in } x, y, z
\end{aligned}
$$

* Presented at the 31st Annual SEG Meeting, Denver, Colorado, November 9, 1961. Manuscript received by the Editor December 12, 1961. Division of Geological Sciences Contribution no. 1059.

$\dagger$ California Institute of Technology, Seismological Laboratory, Pasadena, California. 


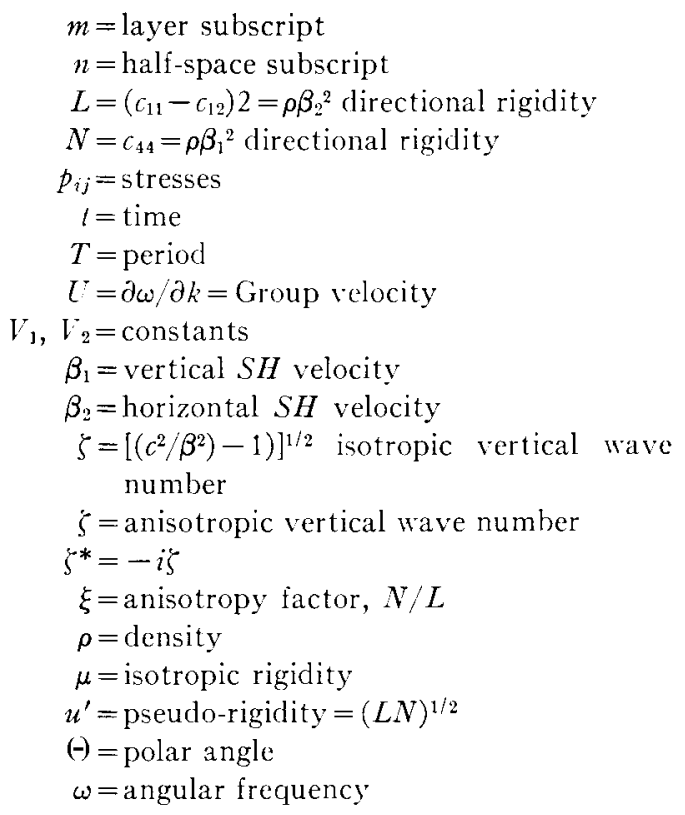

\section{EQUATIONS OF MOTION}

Consider plane waves of angular frequency $\omega$ propagated in the positive $x$-direction with phase velocity, $c$, in a semi-infinite medium composed of $n$ parallel homogeneous transversely isotropic layers. The $n$th layer is an anisotropic half-space. The geometry under consideration and the numbering of the layers and interfaces is illustrated in Figure 1. The following technique is similar to that developed by Haskell (1953) for isotropic layers.

Associated with the $m$ th layer are its density, $\rho_{m}$, thickness, $d_{m}$, and directional rigidities, $N_{m}$ and $L_{m}$. The equation of Love-type motion in the $m$ th layer is

$$
\rho_{m} \frac{\partial^{2} v_{m}}{\partial t^{2}}=N_{m} \frac{\partial}{\partial x} e_{x y}+L_{m} \frac{\partial}{\partial z} e_{y 2},
$$

where

$$
\begin{aligned}
N_{m} & =\left(\frac{c_{11}-c_{12}}{2}\right)_{m}=\left(\rho \beta_{2}^{2}\right)_{m}, \\
L_{m} & =\left(c_{44}\right)_{m}=\left(\rho \beta_{1}{ }^{2}\right)_{m}, \\
\beta_{1} & =\text { vertical } S H \text { velocity, } \\
\beta_{2} & =\text { horizontal } S H \text { velocity, and } \\
v & =\text { horizontal transverse displacement. }
\end{aligned}
$$

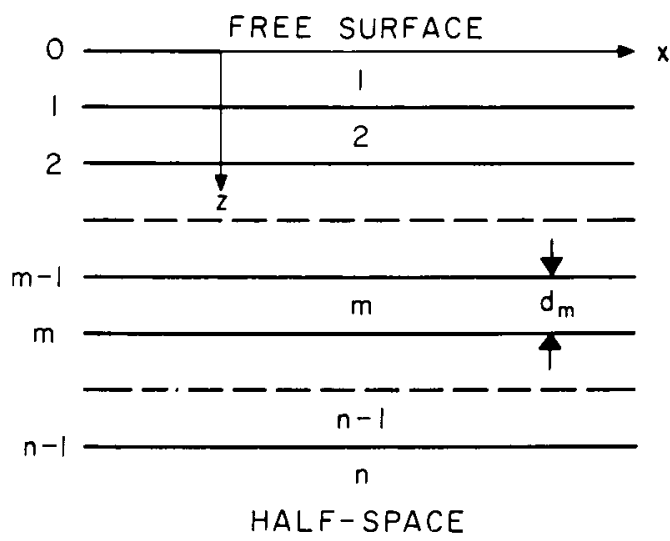

Fig. 1. Geometry of the layered situation under investigation.

The velocity of $S H$ waves satisfies the equation (Stoneley, 1949):

$$
\rho \beta^{2}=l^{2} \Lambda+n^{2} L,
$$

where $l$ and $n$ are the direction cosines from the $x$ and $z$ axes. For isotropic media, $L=N=\mu$ and $\rho \beta^{2}=\mu$ since $l^{2}+n^{2}=1$.

Figure 2 shows schematically the velocity surface of a wave satisfying this equation.

The strains are

$$
e_{x y}=\frac{\partial v}{\partial x} \text { and } e_{y z}=\frac{\partial v}{\partial z},
$$

from which we obtain

$$
\rho_{m} \frac{\partial^{2} v_{m}}{\partial t^{2}}=N_{m} \frac{\partial^{2} v_{m}}{\partial x^{2}}+L_{m} \frac{\partial^{2} v_{m}}{\partial z^{2}} .
$$

This is the displacement equation of motion for $S H$-type motion in transversely isotropic material.

The plane wave solution of equation (4) for an anisotropic layer is

$$
\begin{aligned}
& u=w=0, \\
& v=\left[V_{1} e^{-i k \zeta z}+V_{2} e^{i k \zeta z}\right] e^{i(\omega t-k x),}
\end{aligned}
$$

where $V_{1}$ and $V_{2}$ are constants.

Substitution into the equation of motion yields

$$
\rho \omega^{2}=N k^{2}+L k^{2} \zeta^{2}
$$

or

$$
\zeta=\left(\frac{N}{L}\right)^{1 / 2}\left(\frac{c^{2}}{\beta_{2}{ }^{2}}-1\right)^{1 / 2}
$$




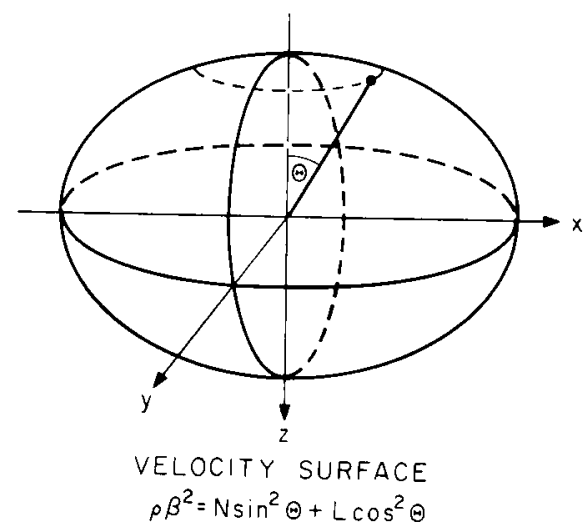

FIG. 2. Velocity surface of $S H$ waves in a transversely isot ropic medium.

\section{BOUNDARY CONDITIONS}

The boundary conditions to be satisfied are that the transverse component of displacement, $v$, and the transverse shear stress, $p_{y z}$, be continuous at each interface.

The transverse shear stress is

$$
\begin{aligned}
p_{y z} & =L \frac{\partial \tau^{\prime}}{\partial z} \\
& =i k \zeta L\left[-V_{i} e^{-i k \zeta z}+V_{2} e^{i k \zeta z}\right] e^{i(\omega t-k \cdot r)} .
\end{aligned}
$$

Following Haskell, we will relate the displacement-stress vectors at the bottom and top of each layer. This relation plus the interface and infinity conditions are sufficient to determine motion at depth in terms of the surface conditions.

Taking the origin of $z$ at the $(m-1)$ th interface we have, at this interface,

$$
(\dot{v} / c)_{m-1}=i k\left(V_{1}+V_{2}\right)_{m}
$$

and

$$
\left(p_{z y}\right)_{m-1}=i k L_{m} \zeta_{m}\left(-V_{1}+V_{2}\right)_{m},
$$

where $c=\omega / k$ is the phase velocity and is the same for all layers.

At the $m$ th interface,

$$
(\dot{v} / c)_{m}=i k\left(V_{1} e^{-i k \zeta_{m} d_{m}}+V_{2} e^{i k \zeta_{m} d_{m}}\right)
$$

and

$$
\begin{aligned}
& \left(\dot{p}_{z y}\right)_{m} \\
& \quad=i L_{m} k \zeta_{m}\left(-V_{1} e^{-i k \zeta_{m} d_{m}}+V_{2} e^{i k \zeta_{m} d_{m}}\right) .
\end{aligned}
$$

By eliminating $V_{1}$ and $V_{2}$ between equations
(8) and (9) we obtain:

$$
\begin{aligned}
(i / c)_{m}= & (j / c)_{m-1} \cos k \zeta_{m} d_{m} \\
& +\frac{i}{L_{m} \zeta_{m}}\left(p_{z y}\right)_{m-1} \sin k \zeta_{m} d_{m}
\end{aligned}
$$

and

$$
\begin{gathered}
\left(p_{z v}\right)_{m}=i L_{-m} \zeta_{m}(i / c)_{m-1} \sin k \zeta_{m} d_{m} \\
+\left(p_{z y}\right)_{m-1} \cos k \zeta_{m} d_{m} .
\end{gathered}
$$

This is a relation between the stress-displacement vectors at the top and bottom of the $m$ th layer and can be written:

$$
\left(i^{\prime}, c, p_{z y}\right)_{m}=a_{m}\left(\dot{i} / c, p_{z y}\right)_{m-1},
$$

where $a_{m}$ is the matrix:

$$
\begin{aligned}
a_{m} & =\cos k \zeta_{m} d_{m} \\
& {\left[\begin{array}{cc}
1 & \frac{i}{L_{m} \zeta_{m}} \tan k \zeta_{m} d_{m} \\
i L_{m} \zeta_{m} \tan k \zeta_{m} d_{m} & 1
\end{array}\right] . }
\end{aligned}
$$

By repeated application we have:

$$
\begin{aligned}
\left(\dot{v} / c, p_{z y}\right)_{n-1} & =a_{n-1} a_{n-2} \cdots a_{1}\left(\dot{v} / c, p_{z y}\right)_{0} \\
& =A\left(\dot{v} / c, p_{z y}\right)_{0}
\end{aligned}
$$

or

$$
\begin{aligned}
& (\dot{v} / c)_{n-1}=A_{11}(\dot{v} / c)_{0}+A_{12}\left(p_{z y}\right)_{0}, \\
& \left(p_{z y}\right)_{n-1}=A_{21}(\dot{v} / c)_{0}+A_{22}\left(p_{z y}\right)_{0},
\end{aligned}
$$

where $A_{i j}$ are the elements of the matrix $A$.

The conditions that must be satisfied for free surface waves with no sources at $z=\infty$ are $\left(p_{z y}\right)_{0}=0$ and $V_{2 n}=0$. This gives

$$
A_{21}=-L_{n} \zeta_{n} A_{11} .
$$

This is the Love-wave dispersion equation for layered anisotropic media.

In the two-layer case, $A=a_{1}$ and equation (16) reduces to

$$
\tan k \zeta_{1} d_{1}=-i \frac{L_{2} \zeta_{2}}{L_{1} \zeta_{1}}
$$

This in turn reduces to the familiar isotropic form when $N=L=\mu$. 


\section{DISCUSSION OF SOLUTION}

Although Love waves are surface waves, equation (16) contains body-wave information. The only $S H$ body-wave information we have discarded in arriving at our solution is the wave leaving the last layer in the direction of positive infinity which, for surface measurements, does not concern us.

In the long-wave limit each $a_{m}$ becomes

$$
a_{m}=\left[\begin{array}{ll}
1 & 0 \\
0 & 1
\end{array}\right], \quad m=1,2, \cdots, n-1 .
$$

Therefore,

$$
A=\coprod_{1}^{n-1} a_{m}=\left[\begin{array}{ll}
1 & 0 \\
0 & 1
\end{array}\right],
$$

and the period equation becomes

$$
L_{n} \zeta_{n}=0
$$

or

$$
c=\boldsymbol{\beta}_{n} .
$$

Therefore, as $k d_{m} \rightarrow 0, m=1,2, \cdots, n-1$, $c \rightarrow \boldsymbol{\beta}_{n}$, the shear velocity in the half-space.

For the short-wave limit, put $\zeta_{m}=i \zeta_{m}{ }^{*}$ to obtain

$$
\zeta_{m}^{*}=\left(\frac{I}{L}\right)^{1 / 2}\left(1-\frac{c^{2}}{\beta_{m}^{2}}\right)^{1 / 2} .
$$

Then,

$a_{m}=\cosh k \zeta_{m}^{*} d_{m}$

$$
\cdot\left[\begin{array}{cc}
1 & -\frac{\tanh \zeta_{m}{ }^{*} k d_{m}}{L_{m} \zeta_{m}} \\
-L_{m} \zeta_{m} \tanh k \zeta_{m}{ }^{*} d_{m} & 1
\end{array}\right] .
$$

As $d_{m} \rightarrow 0, m=2,3, \cdots, n-1$,

$$
a_{m} \rightarrow\left[\begin{array}{ll}
1 & 0 \\
0 & 1
\end{array}\right]
$$

and

$$
A=\cosh k \zeta_{0}{ }^{*} d_{0}
$$

$$
\cdot\left[\begin{array}{cc}
1 & -\frac{\tanh \zeta_{0}{ }^{*} k d_{0}}{L_{0} \zeta_{0}} \\
-L_{0} \zeta_{0} \tanh k \zeta_{0}{ }^{*} d_{0} & 1
\end{array}\right]
$$

giving the period equation:

$$
\tan k \zeta_{0} d_{0}=\frac{L_{n} \zeta_{n}^{*}}{L_{0} \zeta_{0}{ }^{*}}
$$

as $c \rightarrow \beta_{0}, \zeta_{0}{ }^{*} \rightarrow 0$, and $k \zeta_{0} d_{0}=0, \pi / 2,3 \pi / 2, \cdots$. Therefore, as $d_{0} \rightarrow \infty, k \zeta_{0} d_{0}$ remains finite and there are an infinite number of modes, or branches, with $c \rightarrow \beta_{0}$ as $k d_{0} \rightarrow \infty$.

Real roots of the general period equation (16) exist only in the range $\beta_{1}<c<\beta_{n}$ and for $L_{m+1} / L_{m}=0$ (a free heterogeneous plate). Complex roots might be expected outside of these regions and would correspond to leaky or nonperfect constructive interference.

In field refraction studies using $S H$ waves, the slopes of travel-time plots would give $\beta_{2}$, but the intercepts would correspond to the vertically traveling wave or $\beta_{1}$.

\section{SOLUTIONS RECAST INTO ISOTROPIC FORM}

For convenience we introduce the following anisotropy factor:

$$
\xi=\frac{X}{L}=\frac{\beta_{2}{ }^{2}}{\beta_{1}{ }^{2}},
$$

which is unity when there is no anisotropy. Then

$$
\begin{aligned}
\zeta & =\xi^{1 / 2}\left(\frac{c^{2}}{\beta_{2}{ }^{2}}-1\right)^{1 / 2} \\
& =\xi^{1 / 2} \tilde{\zeta}
\end{aligned}
$$

in terms of isotropic constants and the anisotropy factor.

Throughout our development, $\zeta$ appears only in the combinations $L \zeta$ and $\zeta d$. In the isotropic case the corresponding terms are $\mu \tilde{\xi}$ and $\xi d$. We can therefore have complete equivalence if we define a pseudo-rigidity and a pseudo-thickness,

$$
\mu^{\prime}=L \xi^{1 / 2}=\left(L_{L} N\right)^{1 / 2},
$$

and

$$
d^{\prime}=\xi^{1 / 2} d=(\mathrm{N} / L)^{1 / 2} d
$$

for each layer. The pseudo-rigidity in the equivalent isotropic case is the geometric mean of the directional rigidities of the anisotropic problem.

The use of these pseudo-parameters makes it possible to use previous Love dispersion numerical data to determine dispersion in layered anisotropic media. For example, the convenient curves, 


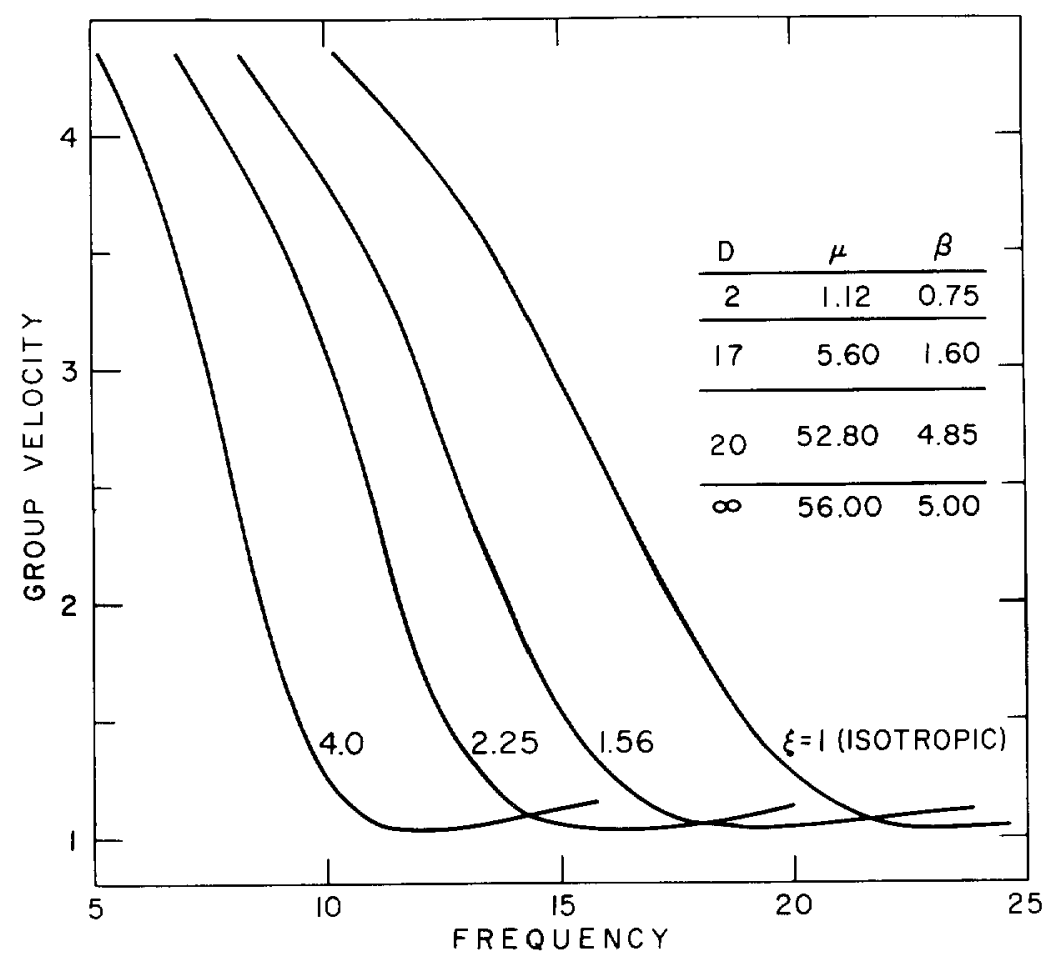

FIG. 3. The effect of a uniform anisotropy on Love wave dispersion in a simple layered structure.

nomograms, and tables of Dorman (1959), Satô (1953), Kanai (1951), and Ewing et al (1957) can be applied to the anisotropic problem. Likewise, computer programs, such as described by Press et al (1961) for calculation of Love-wave dispersion curves in layered isotropic media can be adapted by use of the pseudo-parameters instead of the isotropic parameters to calculate dispersion in layered anisotropic material. Most of the theory developed for isotropic $S H$ and Love-wave propagation, including source and amplitude studies, can be applied directly to the anisotropic problem.

A further simplification results from the fact that Love-wave dispersion is remarkably insensitive to reasonable changes in the rigidity or rigidity gradient which means, if the velocity is unchanged, a lack of sensitivity to density or density gradient. If the rigidity of a whole section is multiplied by a constant factor, such as $\xi^{-1 / 2}$, the dispersion is unchanged since rigidities occur only in ratios. The effect of a uniform anisotropy, then, is to telescope the whole section, which is the same as telescoping the $T$ (period) axis of the dispersion curve. For nonuniform anisotropies the section is telescoped nonuniformily, but the additional effect on rigidities can usually be ignored. Physically, this procedure may be interpreted as changing the travel time in the vertical direction and, therefore, changing the vertical velocity relative to the horizontal, which is just the type of anisotropy we have introduced.

Figure 3 shows the effect of uniform anisotropy on Love waves in a simple layered structure. Note that the frequency for a given velocity is proportional to $\xi^{-1 / 2}$.

\section{TRANSVERSE ISOTROPY AS THE LIMIT OF A LAYERED SOLID}

Postma (1955), and White and Angona (1955) have shown that, in situations of plane stress or infinite wave length, a formation consisting of alternating plane parallel isotropic layers of different properties can be regarded as a homogeneous transversely isotropic system. One would expect this correspondence to hold for wave lengths long compared to the individual layer thicknesses. 
The resulting anisotropy is not as general as that developed in this paper since the inequality $N>L$ must hold for a laminated body, and, of course, the long wave length restriction can be quite severe. To investigate the range of validity of the above correspondence, we will carry out a more general analysis.

Consider a laminated media made up of alternating layers of two different isotropic materials. A section of the material is then composed of doublets, not necessarily identical in component or total thicknesses. The matrix relating conditions at the top and bottom of each doublet is as follows:
The matrices, $a_{m}$ and $b_{m}$, are equal and independent of phase velocity if

$$
Y=\frac{\mu_{m-1} d_{m-1}}{d}+\mu_{m} d_{m}
$$

and

$$
L=\frac{\mu_{m} \mu_{m-1} d}{\mu_{m-1} d_{m}+\mu_{m} d_{m-1}} .
$$

Therefore, a doublet or a series of doublets may be replaced by a single anisotropic layer with the above directional properties. These relations are

$$
\begin{aligned}
& b_{m}=a_{m-1} a_{m} \\
& =\cos k \tilde{\zeta}_{m-1} d_{m-1} \cos k \zeta_{m} d_{m}\left[\begin{array}{ccc}
1 & \\
1 & \frac{1}{\mu_{m-1} \tilde{\zeta}_{m-1}} & \tan k \zeta_{m-1} d_{m-1} \\
i \mu_{m-1} \hat{\zeta}_{m-1} \tan k \hat{\zeta}_{m-1} d_{m-1} & 1
\end{array}\right] \\
& \times\left[\begin{array}{cc}
1 & \frac{1}{\mu_{m} \xi_{m}} \tan k \tilde{\zeta}_{m} d_{m} \\
i \mu_{m} \zeta_{m} \tan k \tilde{\zeta}_{m} d_{m} & 1
\end{array}\right] \\
& =\cos k \tilde{\zeta}_{m-1} d_{m-1} \cos k \tilde{\zeta}_{m} d_{m}
\end{aligned}
$$

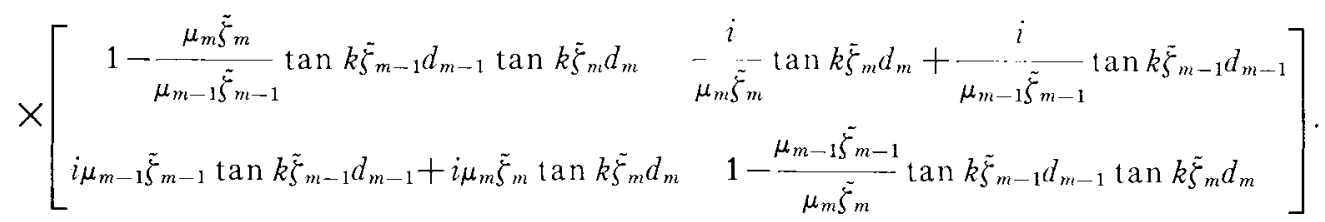

Consider now wave lengths long compared to any doublet thickness. Then,

identical with those given by Postma (1955) from purely static considerations. This analysis may

$$
b_{m}=\left[\begin{array}{cc}
1 & i k\left(\frac{d_{m}}{\mu_{m}}+\frac{d_{m-1}}{\mu_{m-1}}\right) \\
i k\left(\mu_{m-1} \xi_{m-1}^{2} d_{m-1}+\mu_{m} \tilde{\zeta}_{m}^{2} d_{m}\right) & 1
\end{array}\right]
$$

to order $k \zeta d$. The first neglected term is of order $(k \tilde{\zeta} d)^{2}$.

To the same order, for a single anisotropic layer,

$$
a_{m}=\left[\begin{array}{cc}
1 & \frac{i d_{m}}{L} \\
k i N_{m} \tilde{\xi}_{m}^{2} d_{m} & 1
\end{array}\right]
$$

be extended in an obvious way to layered media with more than two components. For long wave lengths, the whole near-surface section may be replaced by an equivalent anisotropic layer. This considerably reduces computational labor for surface-wave studies on multilayered media.

For a layered solid, the directional rigidities, $L$ and $N$, are, respectively, the harmonic and 
arithmetic means of the component rigidities. The effective density is the arithmetic mean of the individual densities. This procedure replaces a layered isotropic structure with a single anisotropic layer. These two structures are equivalent for long wave rengths. If we now form the geometric mean of the directional rigidities and change the thickness of the layer as shown in the previous section, we can replace the single anisotropic layer by an equivalent single isotropic layer.

This analysis shows the correct way to average properties when several isotropic layers are replaced by one. Instead of averaging velocities, as has been common practice, we must form the geometric mean of the arithmetic and harmonic means of the rigidities and modify the thickness.

For a material composed of $N$ laminations, each of different rigidity, $\mu_{i}$, and thickness, $d_{i}$, the equivalent single isotropic layer has the following properties:

$$
\begin{gathered}
\mu^{\prime}=\left\{\frac{\left.\left(\prod_{1}^{N} \mu_{i}\right)\left(\sum_{1}^{N} d_{i} \mu_{i}\right)\right|^{1 / 2}}{\sum_{1}^{N}\left(d_{i} \prod_{1}^{N} \mu_{j}\right)}\right\}_{j \neq i}^{N}, \\
d^{\prime}=\left\{\frac{\left(\sum_{1}^{N} d_{i} \mu_{i}\right) \sum_{1}^{N} d_{i}\left(\prod_{1}^{N} \mu_{j \neq i}\right)}{\left(\prod_{1}^{N} \mu_{i}\right)}\right\}^{1 / 2}
\end{gathered}
$$

for

$$
k \sum_{1}^{N} d_{i} \ll 1 .
$$

To illustrate the range of validity of the laminated solid approximation and as an independent check on the theory developed in this paper, several numerical examples were computed on the Love-wave dispersion program described by Press et al (1961).

- In the first example, phase velocities were computed for Love-type motion of a laminated, 10 layer plate and for the equivalent homogeneous anisotropic plate. The parameters are taken from Postma (1955) and are tabulated in Tables 1 and

\begin{tabular}{|c|c|c|c|c|c|}
\hline & \multicolumn{2}{|c|}{$d_{1} / d_{2}$} & \multirow{2}{*}{$\mu$} & \multirow{2}{*}{$\beta$} & \multirow{2}{*}{$\rho$} \\
\hline & I & II & & & \\
\hline 1 & $\frac{1}{3}$ & 3 & 2.5 & 3,04 & 2.7 \\
\hline 2 & 1 & 1 & 0.6 & 1.62 & 2.3 \\
\hline
\end{tabular}

Table 1. Parameters for Postma Solids (I and II) in arbitrary consistent units.

Table 2. Parameters for equivalent anisotropic solids.

\begin{tabular}{rccccccccc}
\hline & $D$ & $D^{\prime}$ & $N$ & $L$ & $\mu^{\prime}$ & $\beta_{1}$ & $\beta_{2}$ & $\rho$ & $\xi$ \\
\hline I & 1 & 1.21 & 1.08 & 0.74 & 0.895 & 1.76 & 2.12 & 2.4 & 1.46 \\
II & 1 & 1.20 & 2.03 & 1.40 & 1.684 & 2.32 & 2.79 & 2.6 & 1.45 \\
\hline
\end{tabular}

Table 3. Phase velocity and period for the fundamental Love mode in anisotropic plate $\left(T_{1}\right)$ and laminated plate $\left(T_{2}\right)$.

\begin{tabular}{ccc}
\hline $\mathrm{C}$ & $T_{1}$ & $T_{2}$ \\
\hline 5.0 & 1.032 & 1.029 \\
4.6 & 1.011 & 1.009 \\
4.2 & 0.983 & 0.981 \\
3.8 & 0.946 & 0.943 \\
3.4 & 0.892 & 0.888 \\
3.0 & 0.806 & 0.804 \\
2.6 & 0.660 & 0.659 \\
\hline
\end{tabular}

2 under Postma Solid I. The results are shown in Table 3. The difference in period is about 2.5 percent at a wave length corresponding to about ten times the thickness of the largest individual layer. The difference gets more pronounced for shorter wave lengths and would get smaller if the laminations were made smaller.

In the second example, a three-layer half-space was constructed as shown in Figure 4 corresponding to a layer of Postma Solid I over a layer of Postma Solid II over an isotropic half-space. Dispersion was computed for each surface layer split into 20 laminations and then into 40 laminations, and also for the equivalent anisotropic layers. As the number of laminations increases, the dispersion converges to the anisotropic case, as shown in Figure 4.

Figure 5 shows the displacements as a function of depth for cases $A$ and $B$, for three values of the phase velocity. The displacements are normalized to the surface value. The effect of the laminations is to keep the particle motion from dying off so rapidly. The displacements for the laminated case diverge more from the anisotropic case as the wave length gets smaller. 


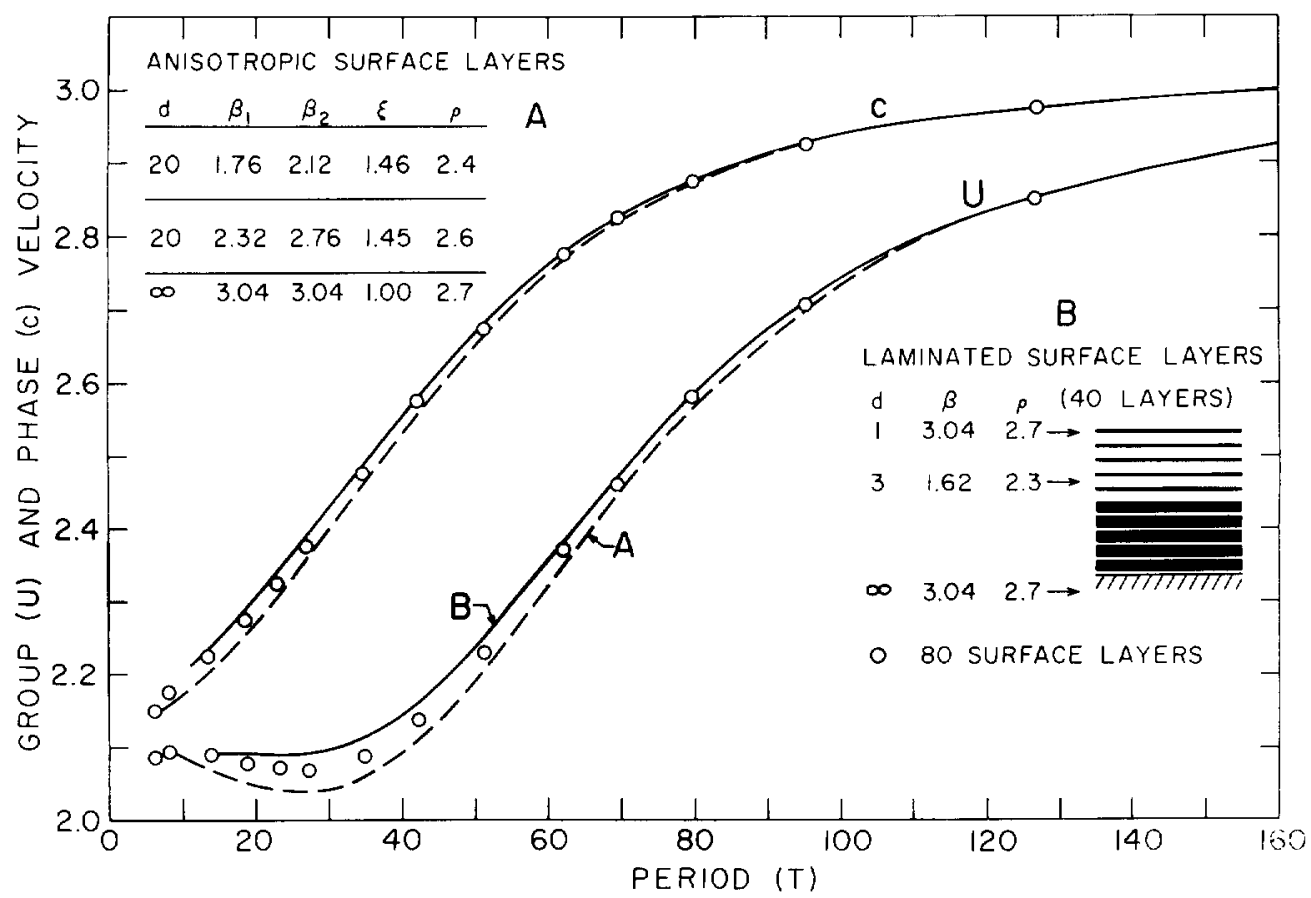

Fic. 4. Dispersion in a system composed of two dnisotropic surface layers overlying an isol ropic halispace, and comparison with the laminated case.

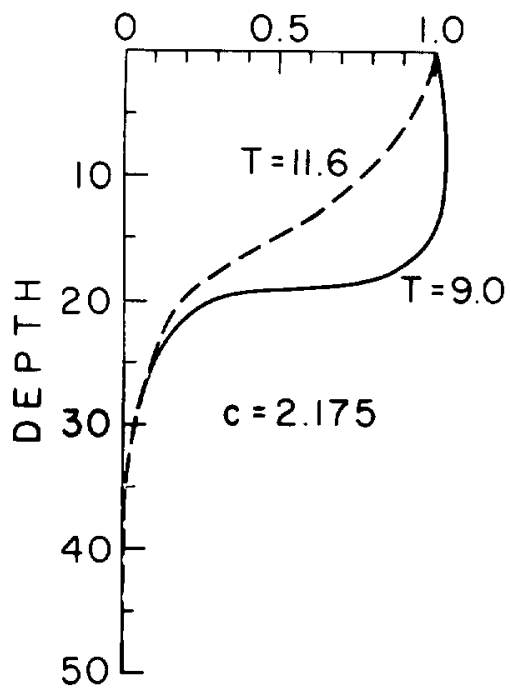

\section{DISPLACEMENTS}

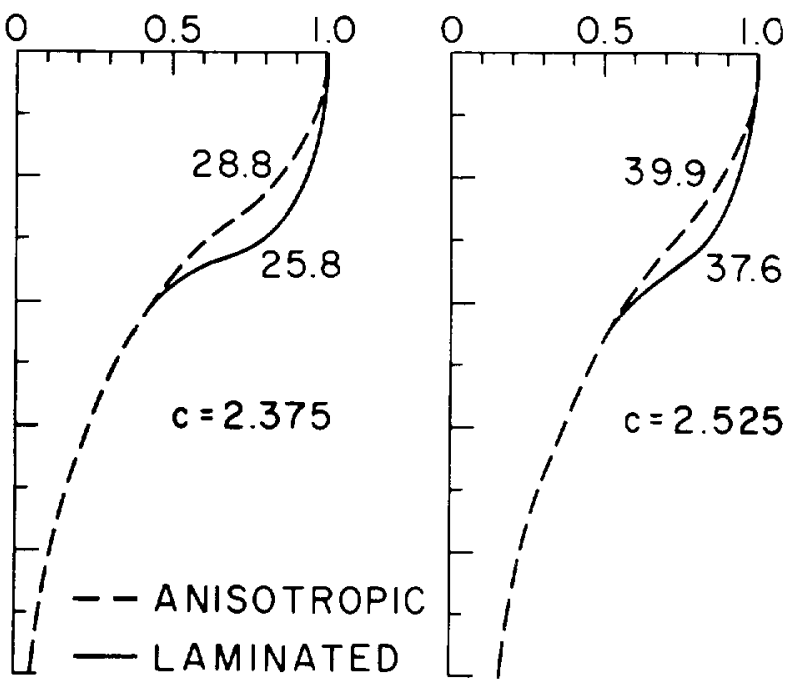

FiG. 5. Horizontal displacements versus depth for cases $A$ (anisotropic) and $B$ (laminated). 


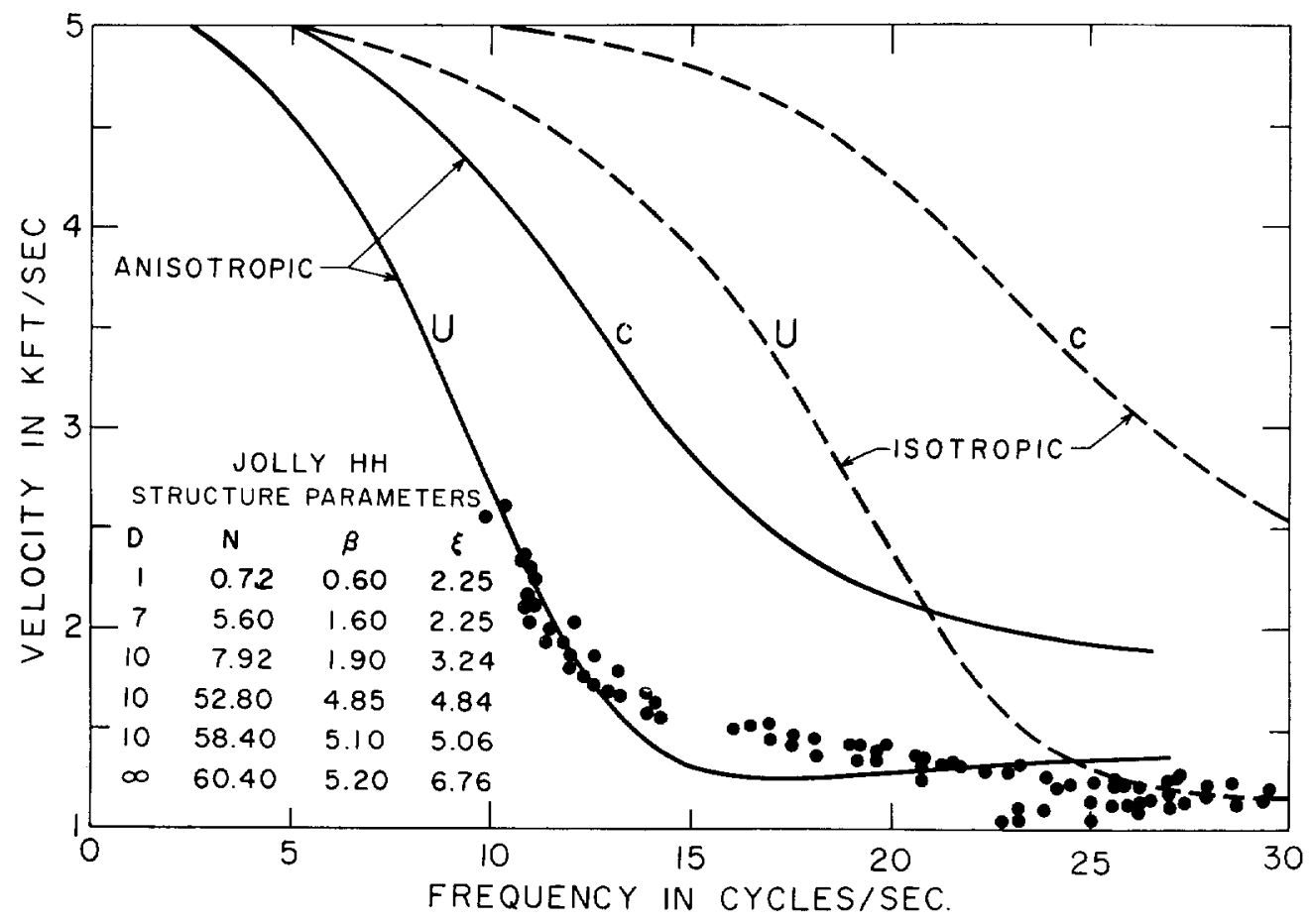

FIG. 6. Isotropic and anisotropic dispersion data compared with field results of Jolly (1956). The structure parameters in the table closely approximate refraction results.

\section{COMPARISON WITH FIELD DATA}

Jolly (1956) in a fundamental field study of shear waves demonstrated the existence of transverse isotropy in a near-surface section of shale. He obtained refraction data for $S H, S V$, and $P$ waves and also some Love-wave data. There was good evidence to indicate that the horizontal $S H$ velocity was approximately twice the vertical velocity. Figure 6 gives Jolly's experimental results and two theoretical curves. The dashed curve is the predicted dispersion using field refraction and reflection data and assuming isotropy. The anisotropic results are given by the solid curves. Structure parameters are given in the table on Figure 6 ; the isotropic curve has $\xi=1$ throughout the section. It is obvious that the assumption of isotropy is not warranted. An increase in anisotropy with depth seems to be indicated although the structure determined is not unique.

\section{ACKNOWLEDGMENTS}

I gratefully acknowledge the support of $\mathrm{Na}$ tional Science Foundation and Standard Oil of
California predoctoral fellowships during the course of this work.

\section{REFERENCES}

Anderson, D. L., 1961, Elastic wave propagation in layered anisotropic media: J. Geoph. Res., v. 66, pp. 2953-2963.

Dorman, J., 1959, Numerical solutions for Love-wave dispersion on a half-space with double surface layer: Geophysics, v. 24, pp. 12-29.

Ewing, W. M., Jardetzky, W. S., and Press F., 1957 , Elastic waves in layered media, New York, McGrawHill Book Co.

Gassman, F., 1951, Elastic waves through a packing of spheres: Geophysics, v. 16, pp. 673-685.

Haskell, N. A., 1953, Dispersion of surface waves on multi-layered media; Bull. Seism, Soc. Am., v. 43, pp. 17-34.

Helbig, K., 1958, Elastiche Wellen in anisotropen Medien: Gerlands Beitrage zur geophysik, v. 67, pp. $177-211,256-288$.

Jolly, R. N., 1956, Investigation of shear waves: Geophysics, v. 21, pp. 905-938.

Kanai, K., 1951, On the group velocity of dispersive surface waves: Bull. Farthquake Res. Inst. (Tokyo), v. 29, pp, $49-60$.

Layet, C., Clement, A. C., Pommier, G., and Buffet, A. Some technical aspects of refraction seismic prospecting in the Sahara: Geophysics, v. 26, pp. 437-446.

Postma, G. W., 1955, Wave propagation in a stratified medium: Geophysics, v. 20, pp. 780-806. 
Press, F., Harkrider, D., and Seafeldt, C. A., 1961, A fast convenient program for computation of surface wave dispersion curves in multilayered media: Bull. Seism, Soc. Am. (in press).

Satô, Y., 1953, Study on surface waves VIII: Bull. Earthquake Res. Inst. (Tokyo), v. 31, pp. 81-87.

Stoneley, R., 1949, The seismological implications of aeolotropy in continental structure: Monthly Notices Roy. Astron. Soc., Geophys. Suppl., v. 5, pp. 222-232.
Uhrig, L. F., and Van Melle, F. A., 1955, Velocity anisotropy in stratified merlia: Geophysics, v. 20 , pp. 774-779.

White, J. E., and Angona, F. A., 1955, Elastic wave velocities in laminated media: J. Acoust. Soc. Am., v. 27, pp. 310-317.

White, J. E., and Sengbush, R. L., 1953, Velocity measurements in near-surface formations: Geophysics, v. 18 , pp. 54-69. 